die Art des Valenzzustandes eines Atoms ablesen kann. Die Aufzeichnung der Formeln erfolgt nach den bisherigen Regeln; z. B.

\begin{tabular}{|c|c|c|c|}
\hline Molekel & Valenzformeln & $\begin{array}{l}\text { Valenzzustände } \\
\text { der Atome }\end{array}$ & Konfiguration \\
\hline $\mathrm{NO}_{2}$ & $\mathrm{~N}^{-}=0$. & $\begin{array}{l}\mathrm{O}(2), \mathrm{p} \\
\mathrm{N}(3), \mathrm{p} \\
\mathrm{O}(2), \mathrm{p}\end{array}$ & Winkel \\
\hline $\mathrm{ClO}_{2}$ & $\cdot \mathrm{Cl}^{\oplus \epsilon}$ & $\begin{array}{l}\mathrm{O}^{-}(1), \mathrm{p} \\
\mathrm{Cl}^{++}(3), \mathrm{p} \\
\mathrm{O}^{-}(1), \mathrm{p}\end{array}$ & Winkel \\
\hline
\end{tabular}

Es sei nochmals darauf hingewiesen, daß man die „neuen" Valenzformeln - wenn man sich einmal mit dem Inhalt der Tab. 3 vertraut gemacht hat - nicht nur ebenso schnell niederschreiben kann wie die älteren Formeln, sondern daß sich die ersteren gegenüber den letzteren als außerordentlich überlegen erweisen. So sind schon z. B. die Ammoniumsalze nur mit den neuen Formeln, welche zwischen der Atombindung und der Ionenbindung unterscheiden, richtig $\mathrm{zu}$ formulieren. In ähnlicher Weise geben nur diese den Unterschied zwischen der „semipolaren“ und der „echten“ Doppelbindung richtig wieder (vgl. z. B. die Formeln der Ketone und Sulfoxyde). Endlich lassen sich nur mit den neuen Formeln Schlüsse auf den räumlichen Bau der Molekeln ziehen.

\title{
Verschiedenartig zusammengesetzte Pentose-Nucleinsäuren der Hefe
}

\section{(2. Mitteilung über Nucleinsäuren*)}

\author{
Von Karl Dimroth und Loth a Jaenicke \\ Aus dem Physiologisch-Chemischen Institut der Universität Marburg (Lahn) \\ (Z. Naturforschg. 5 b, 185-188 [1950]; eingegangen am 28. April 1950)
}

\begin{abstract}
Durch Züchtung von Torula unter verschiedenen Bedingungen konnten Nucleinsäuren erhalten werden, deren Zusammensetzung in bezug auf die beiden Purine Adenin und Guanin von der Stickstoffquelle des Substrats abhängig ist. Durch Hemmung mit Azid wurde die Ausbeute an Nucleinsäure gesteigert und deren Gehalt an Adenin um fast das Doppelte erhöht.
\end{abstract}

$\mathrm{D}$ ie Pentose-Nucleinsäure der Hefe, die man vor der Entdeckung der Desoxypentose-Nucleinsäure in der Hefe ${ }^{1 a}$ meist als „Hefenucleinsäure“ bezeichnete, wird im allgemeinen als der Prototyp der Pentose-Nucleinsäuren überhaupt aufgefaßt und als Naturstoff konstanter Zusammensetzung betrachtet. Nach der auf Leven e ${ }^{1 b}$ zurückgehenden Vorstellung, die von den meisten Lehrbüchern übernommen worden ist, setzt sich eine solche Nucleinsäure aus Tetranucleotid-Bausteinen zusammen, die ihrerseits aus vier, die Basen Guanin, Adenin, Cytosin und Uracil, sowie Ribose und Phosphorsäure enthaltenden Mononucleotiden bestehen. Für diese Vorstellung spricht vor allem die Tatsache, daß es trotz eifrigen Suchens bisher nicht gelungen ist, andere Bruchstücke als die genannten Basen bzw. die sich von ihnen ableitenden Mono-Nucleoside und -Nucleotide zu finden. Andererseits jedoch bestehen auch er-

* 1. Mitteilung: Liebigs Ann. Chem. 566, 206 [1950]. 1a E. C h a r g a f u. St. Z a me $n$ hof, J. Amer. Chem. Soc. 69,.975 [1947].

1b P. A. Levene u. L. W. B a s s, Nucleic Acids, Chemical Catalog Co., New York 1931; P. A. L e ve ne, Biochem. Z. 17, 120 [1909]. hebliche Bedenken gegen die Gültigkeit der Tetranucleotid-Vorstellung. $\mathrm{Gu} \mathrm{ll}$ a $\mathrm{n} \mathrm{d}^{2}$ und $\mathrm{Ch}$ a r g a $\mathrm{ff}$ u. V i s ch e ${ }^{3}$ haben in ausführlichen Diskussionen auf die Mängel hingewiesen: 1. Die analytischen Bestimmungen der 4 Basen ergaben niemals Werte, aus denen ein gesicherter Schluß auf ein äquimolares Verhältnis gezogen werden kann; 2. die Isolierung einheitlicher Tetranucleotid-Bausteine ist niemals gelungen ${ }^{3 a}$. Màn muß allerdings beachten, daß fast alle Untersuchungen an technischen Nucleinsäuren ausgeführt worden sind, deren molekulare Struktur möglicherweise verändert war, daß ferner die quantitativen Bestimmungsmethoden sehr mangelhaft sind, so daß ein einheitliches Bild noch nicht gewonnen werden kann.

Für unsere Untersuchungen über den Nucleinsäure-Stoffwechsel von Hefen haben wir einen aus einer Betriebshefe der Zellstoff-Fabrik 'Waldhof ge-

2 J. M. Gulland u. Mitarbb., Annu. Rev. Biochem. 14, 175 [1945].

3 E. C h a rgaff u. E. Vis cher, Annu. Rev. Biochem. 17, 201 [1948]; E. Vis cher u. E. C hargaff, J. biol. Chemistry 176, 715 [1948]; 177, 405 [1949].

a F. G. F i s c h e r, Z. angew. Chem. 56, 329 [1943]. 


\begin{tabular}{|c|c|c|c|c|c|c|}
\hline \multirow{2}{*}{$\begin{array}{l}\text { Stickstoffquelle } \\
\text { je } 1 / 20 \mathrm{Mol}\end{array}$} & \multirow{2}{*}{$\begin{array}{l}\text { Verdoppelungs- } \\
\text { zeit [min] }\end{array}$} & \multirow{2}{*}{$\begin{array}{l}\text { Nucleinsäure } \\
\% \text { der Trocken- } \\
\text { substanz }\end{array}$} & \multicolumn{3}{|c|}{$\begin{array}{l}100 \text { mg Nucleinsäure } \\
\text { ergaben an Purinen }\end{array}$} & \multirow{2}{*}{$\begin{array}{l}\text { Verhältnis } \\
\frac{\text { Guanin }}{\text { Adenin }}\end{array}$} \\
\hline & & & Gesamt & Guanin & Adenin & \\
\hline Ammoniak . . . . & 150 & 3,5 & $\begin{array}{l}30,5 \\
30,0\end{array}$ & $\begin{array}{l}14,5 \\
15,0\end{array}$ & $\begin{array}{l}13,5 \\
14,0\end{array}$ & $\begin{array}{l}1,07 \\
1,07\end{array}$ \\
\hline Glykokoll . . . . . & 110 & 7,0 & 28,5 & 14,0 & 14,0 & 1,00 \\
\hline Serin . . . . . & 170 & 7,2 & $\begin{array}{l}34,6 \\
23,9\end{array}$ & $\begin{array}{l}18,4 \\
10,9\end{array}$ & $\begin{array}{l}15,4 \\
10,0\end{array}$ & $\begin{array}{l}1,19 \\
1,09\end{array}$ \\
\hline Adenin . . . . . & 210 & 7,0 & $\begin{array}{l}26,2 \\
23,2\end{array}$ & $\begin{array}{r}11,2 \\
9,6\end{array}$ & $\begin{array}{l}14,2 \\
12,2\end{array}$ & $\begin{array}{l}0,79 \\
0,79\end{array}$ \\
\hline Guanin . . . . . & 255 & 5,7 & $\begin{array}{l}41,3 \\
38,3\end{array}$ & $\begin{array}{l}22,7 \\
19,8\end{array}$ & $\begin{array}{l}15,3 \\
13,2\end{array}$ & $\begin{array}{l}1,44 \\
1,50\end{array}$ \\
\hline Ammoniak/Azid . . & 2400 & 5,4 & $\begin{array}{l}25,5 \\
21,7\end{array}$ & $\begin{array}{l}9,1 \\
7,7\end{array}$ & $\begin{array}{l}14,5 \\
13,1\end{array}$ & $\begin{array}{l}0,63 \\
0,59\end{array}$ \\
\hline Glykokoll/Azid . . & - & 7,9 & 35,9 & 15,5 & 20,3 & 0,77 \\
\hline Ammoniak $/ \mathrm{BeCl}_{2}$. & 230 & 2,0 & 33,5 & 17,0 & 14,8 & 1,15 \\
\hline
\end{tabular}

Tab. 1.

züchteten Torula-Stamm unter konstanten äußeren Bedingungen (Temperatur, Wasserstoffionen-Konzentration, Belüftung und Belichtung) auf Nährsubstraten wachsen lassen, die sich nur dadurch unterschieden, daß der für das Wachstum notwendige Stickstoff in Form verschiedener Stickstoff-Verbindungen gegeben wurde. Wir fanden zunächst eine spezifische Abhängigkeit der Vermehrungsgeschwindigkeit von der jeweiligen Stickstoffquelle. Wie noch mitgeteilt werden soll, ist der Nucleinsäuregehalt der Hefen ermittelt durch Extraktion mit heißer 10-proz. NaClLösung - unter diesen verschiedenen Wachstumsbedingungen sehr stark von der Art der Stickstoffquelle abhängig und durchaus nicht immer der Vermehrungsgeschwindigkeit proportional; es gehen hier also offenbar Eiweißbildung und Nucleinsäureproduktion nicht einander parallel, wie man es vielleicht nach den Arbeiten von Casperss on ${ }^{4}$ erwarten sollte.

Als wir die Nucleinsäuren der auf verschiedenen Stickstoffsubstraten gewachsenen Hefen näher untersuchten, fanden wir, daß auch ihre Zusammensetzung von der Stickstoffquelle und den Wachstumsbedingungen abhängig ist. Wir beschränkten uns zunächst auf die Bestimmung der Purinbasen Adenin und Guanin nach der Hydrolyse der Nucleinsäure mit Methanol/Salzsäure ${ }^{5}$.

Während die Hefe bei Fütterung mit Ammoniumsulfat oder den Aminosäuren Glykokoll und Serin

4 T. C a s p e r s s o n, Naturwiss. 29, 33 [1941].

5 G. Hunter u. I. H ly nka, Biochemic. J. 31, 486 [1937]. trotz recht verschiedener Wachstumsgeschwindigkeit und Gesamt-Nucleinsäure-Ausbeute eine Nucleinsäure liefert, bei der das Verhältnis Guanin : Adenin nahezu gleich 1 ist, bildet sie beim Wachsen mit Adenin eine Nucleinsäure, die erheblich mehr Adenin eingebaut enthält; umgekehrt entsteht beim Wachsen mit Guanin eine Nucleinsäure mit viel mehr eingebautem Guanin. Daß es sich hierbei nicht etwa um eine aus der Nährlösung stammende Verunreinigung der Nucleinsäure mit dem betreffenden Purin handelt, kann dadurch gezeigt werden, daß man in Farallelversuchen der Hefe vor der Extraktion der Nucleinsäuren freies Guanin bzw. Adenin zusetzt: Das ungebundene Purin wird nicht mit der Nucleinsäure gefällt, sondern verbleibt in Lösung.

Einen erheblichen Anstieg des Adeningehaltes der Hefe-Nucleinsäure findet man, wenn man die Hefe mit Glykokoll oder Ammoniak als Stickstoffquelle wachsen läßt und das Wachstum noch vor dem Verbrauch des Nährsubstrates mit Natriumazid hemmt. Wie Abb. 1 zeigt, wird nach Zusatz von $1 \mathrm{mMol}$ Natriumazid das Wachstum sofort verlangsamt, während gleichzeitig das $p_{\mathrm{H}}$ auf 3,4 bis 3,7 sinkt. Noch bevor aller Zucker verheft ist, hört das Wachstum auf. Der Nucleinsäuregehalt der Hefe steigt deutlich an: Bei Fütterung mit Ammoniak und Hemmung mit Azid vom Normalwert $3,4-3,6 \%$ auf $5,4 \%$, bei Fütterung mit Glykokoll und Hemmung mit Azid von $7,0 \%$ auf $7,9 \%$. Wird die im Hemmversuch gewachsene Hefe in frische Nährlösung überimpft, wächst sie normal weiter; eine im Hemmversuch auftretende rote Färbung verschwindet wieder. Es ist 
möglich, daß das Azid durch spezifische Hemmung der sauren Phosphatase wirkt ${ }^{6}$. Versucht man die alkalische Phosphatase durch Berylliumchlorid zu hemmen ${ }^{7}$, findet nur eine geringe Verlangsamung des Hefewachstums statt. Die Ausbeute an Nucleinsäure ist jedoch mit $2 \%$ gegenüber $3,4-3,6 \%$ unter Standardbedingungen (Ammoniakfütterung, $p_{\mathrm{H}} 5,6$ bis 6,$0 ; 32^{\circ} \mathrm{C}$; diffuses Tageslicht) deutlich verringert. Ihre Zusammensetzung ist nicht wesentlich verändert.

Die Ergebnisse der Purin-Analysen sind in Tab. 1, berechnet auf $100 \mathrm{mg}$ bei $60^{\circ}$ im Vakuum getrocknete Nucleinsäure, zusammengestellt.

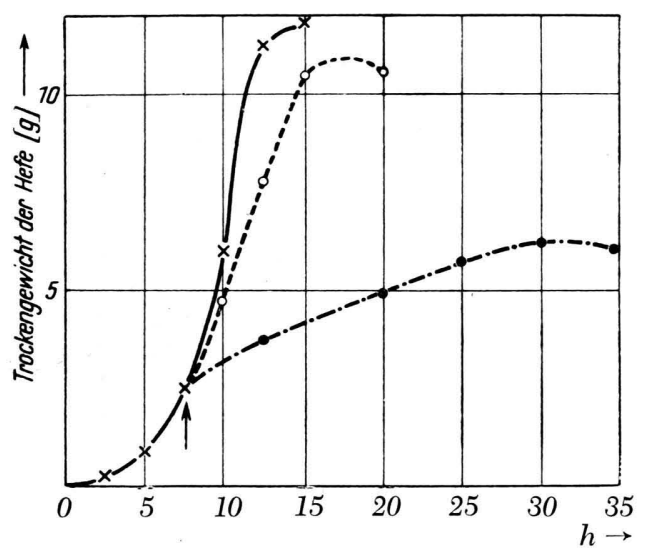

Abb. 1. Wachstumsgeschwindigkeit der Hefe auf Rohrzucker-Ammoniak unter Normalbedingungen (- ohne Hemmstoff) und nach Zusatz von $\mathrm{BeCl}_{2}(----)$ bzw. $\mathrm{NaN}_{3}$ (-.--). Der senkrechte Pfeil gibt den Zeitpunkt für den Zusatz des Hemmstoffs an.

Die Ergebnisse zeigen, daß es möglich ist, durch Variation der Wachstumsbedingungen Hefen zu züchten, deren Nucleinsäuren verschieden zusammengesetzt sind. Man muß offenbar die Nucleinsäuren als Naturstoffe auffassen, deren Aufbau und Zusammensetzung viel größeren Veränderungsmöglichkeiten unterworfen sein können, als man es bisher annahm. Mit den Vorstellungen eines Aufbaues der Nucleinsäuren aus Tetranucleotid-Bausteinen lassen sich unsere Ergebnisse nicht erklären. Es scheint uns vielmehr wahrscheinlich, daß sich die Nucleinsäuren in ähnlicher Weise durch Kombination einzelner Nucleotide aufbauen wie die Eiweißkörper durch Aneinanderfügen einzelner Aminosäuren. Die Bausteinanalyse liefert nur statistische Mittelwerte. Die sehr großen Schwierigkeiten, die sich heute noch

6 C. E. Clif to n, Advanc. Enzymol. VI, 269 [1946].

7 F. W. Klemperer, J. M. Miller u. C. J. Hill, J. biol. Chemistry 180, 281 [1949]. einer quantitativen Analyse der Nucleotide entgegenstellen, machen es verständlich, daß man kleinere Unterschiede in der Zusammensetzung noch nicht mit Sicherheit erkennen kann. In den von uns geschilderten Beispielen sind die Unterschiede aber so groß, d: $ß$ sie außerhalb der Fehlergrenze der Bestimmungsmethode liegen. Der starke Anstieg des Adenins bei Hemmung mit Azid kann vielleicht auf einer kompensatorischen Überproduktion des gehemmten AdenosinNucleotids beruhen und anzeigen, daß dieses in die Nucleinsäure eingebaut ist.

\section{Beschreibung der Versuche}

Die Hefe wird in 2-l-Rundkolben im Wasserthermostaten bei $32^{\circ}$ gezüchtet. Die Zuchtkolben sind mit Gaseinleitungsrohr mit G 2 Fritte, Antimon/ges. Kalomel-Elektroden und Einfüllstutzen zum Nachfüllen und Probenehmen versehen. Die Grundnährlösung enthält

$\left.\begin{array}{clc}15 & \text { g Rohrzucker } & \mathrm{FeSO}_{4} \\ 1 & \text { g Kaliumchlorid } & \mathrm{ZnSO}_{4} \\ 0,7 \text { g Magnesiumsulfat } \cdot 7 \mathrm{H}_{2} \mathrm{O} \mathrm{MnSO}_{4} \\ 1 \text { g prim. Kaliumphosphat } & \mathrm{CuSO}_{4}\end{array}\right\} \begin{gathered}\text { je 1 Tropfen } \\ \text { einer } \\ \text { 0,3 g sek. Natriumphosphat }\end{gathered}$

in $1 l$ Leitungswasser gelöst. Dann werden der Lösung $0,05 \mathrm{Mol}$ gebundener Stickstoff in Ferm von A m mo nsulfat bzw. Glykokoll, Alanin, Serin, AdeninHydrochlorid oder Guanin-Hydrochlorid zugesetzt und das $p_{\mathrm{H}}$ auf 5,8 eingestelit; Kolben und Nährlösung werden im Dampftopf sterilisiert. Man impft in $500 \mathrm{~cm}^{3}$ Nährlösung $100 \mathrm{mg}$ Hefe-Reinkultur aus Schräg-Agar-Röhrchen ein und verheft unter kräftigeni Durchleiten von steriler Luft $(100 l / \mathrm{h})$. Das $p_{\mathrm{H}}$ der Lösung wird elektrometrisch gemessen und durch Zugabe von $\mathrm{NaOH}$ auf 5,8-6,2 gehalten. Zur Kontrolle des Wachstums werden im Abstand von $2 \mathrm{~h}$ Proben steril entnommen und in einer Zählkammer ausgezählt. Durch Vorversuche war festgestellt worden, daß 8000 Mill. Zellen etwa $1 \mathrm{~g}$ Hefe mit $20 \%$ Trockensubstanzgehalt entsprechen. Nach Vermehrung auf etwa $1 \mathrm{~g}$ Hefe gibt man weitere $500 \mathrm{~cm}^{3}$ Nährlösung $\mathrm{zu}$ und gegebenenfalls das $\mathrm{N}$ a tri u mazid (65 mg) oder B eryllium chlorid (152 mg). Der Ansatz wird zu Ende verheft, was man an dem Stagnieren und schließlichen Abnehmen der Ze!l erkennt. Man zentrifugiert die milchige Hefesuspension ab, wäscht die Hefe mit physiologischer Kochsalzlösung und extrahiert die Nucleinsäure. Hierzu wird die Hefe mit dem gleichen Volumen 20-proz. NaCl-Lösung verrührt und im Zentrifugenglas $10 \mathrm{~h}$ bei $90-95^{\circ}$ gehalten. Dann wird zentrifugiert und die klare gelbe Lösung tropfenweise mit 5- $n$. $\mathrm{HCl}$ versetzt, bis bei $p_{\mathrm{H}}=2$ die rohe Nucleinsäure flockig ausfällt. Sie wird abzentrifugiert, mit Methanol, Aceton und Äther gewaschen und $3 \mathrm{~h}$ im Vakuum bei $60^{\circ}$ getrocknet. Die fast weiße, pulvrige Nucleinsäure wird in dem 4-fachen Vol. absol. Methanol suspendiert und in 25- $\mathrm{cm}^{3}$-Birnenkolben mit Gaseinleitungsrohr und Rückflußkühler hydrolysiert, indem man bei $50^{\circ}$ unter sorgfältigem Feuchtigkeitsausschluß trockenen Chlorwasserstoff einleitet. Die Nuclein- 
säure löst sich innerhalb von etwa 30 min und die PurinHydrochloride beginnen auszufallen. Das dunkle Hydrolysat wird über Nacht unter Ausschluß von Feuchtigkeit im Eisschrank aufbewahrt; dann werden die Hydrochloride auf einer $\mathrm{G}_{3}$-Mikronutsche abgesaugt. Man löst sie in der 10-fachen Menge Wasser, filtriert nach Zusatz von wenig Norit-Kohle und versetzt mit 1/5 Vol. konz. HCl. Das Guanin-Hydrochlorid läßt man im Eisschrank auskristallisieren; es wird aus Wasser umkristallisiert und gewogen. Die Reinheit wird mit dem Beckman-Spektralphotometer geprüft (Max. $250 \mathrm{~m} \mu, \varepsilon=11800$ ). Die Mutterlaugen werden im Vakuum bis zur Kristallisation eingeengt, $6 \mathrm{~h}$ im Eisschrank aufbewahrt und das Adenin-Hydrochlorid abfiltriert. Die Restlaugen ergaben noch eine weitere kleine Menge Adenin-HCl. (Max. $260 \mathrm{~m} \mu, \varepsilon=16200$ ). Zur Kontrolle wurde dies Verfahren mit einer Nuclein- säure erprobt, deren Zusammensetzung uns aus größeren Ansätzen bekannt war. Aus $100 \mathrm{~g}$ wurden erhalten: 12,7 g Guanin-HCl und 10,5 g Adenin- $\mathrm{HCl}$; im Mikroversuch mit $200 \mathrm{mg}$

24,8 mg Guanin- $\mathrm{HCl}$ und 23,1 mg:Adenin- $\mathrm{HCl}$, $26,6 \mathrm{mg}$ Guanin- $\mathrm{HCl}$ und 22,4 mg Adenin- $\mathrm{HCl}$, $23,9 \mathrm{mg}$ Guanin-HCl und 22,0 mg Adenin-HCl, durchschnittl. 25,2 mg ( $\pm 6 \%$ ) Guanin- $\mathrm{HCl}$ und $22,5 \mathrm{mg}( \pm 3 \%)$ Adenin- $\mathrm{HCl}$.

In den Mutterlaugen der Purin-Hydrochloride befinden sich die Pyrimidin-Riboside, deren Aufspaltung mit Ameisensäure ${ }^{3}$ sehr verlustreich ist und unter teilweiser Desaminierung verläuft. Die Pyrimidin-Bestimmungen sind noch so wenig befriedigend, daß sie sich nicht für unsere Untersuchungen anwenden ließen.

\title{
Zur Auswertung chemischer Versuchsergebnisse nach der Methode der kleinsten Quadrate (Mit einem Beispiel der Fettspaltung)
}

\author{
Von H. WolF* \\ (Z. Naturforschg. 5 b, 188-190 [1950]; eingegangen am 28. Februar 1950)
}

W Till man aus empirischen Versuchsergebnissen die Parameter einer thecretisch zumeist bekennten $\mathrm{Ge}$ setzmäßigkeit ermitteln, so wird man im allgemeinen zu streuenden Ergebnissen gelangen, weil die Meßdaten mit unvermeidbaren Meßfehlern behaftet sind; und zwar tritt diese Mehrdeutigkeit der Ergebnisse insbesondere dann in Erscheinung, wenn die Messungen in größerer Anzahl ausgeführt wurden, als zur eindeutigen Bestimmung der gesuchten „Konstanten“ unbedingt erforderlich gewesen wäre.

Zur Behebung dieses Mißstandes hat man nach einem bekannten Grundsatz der Methode der kleinsten Quadrate die Bestimmung so durchzuführen, daß die gesuchten Werte die Eigenschaft von wahrscheinlichsten oder „plausibelsten“ Größen besitzen. Denkt man sich zu diesem Zweck an jedem Meßwert eine Verbesserung $v$ angebracht, so führt dies zur Gau $ß$ schen Minimumsbedingung, daß

$$
\sum_{i=1}^{\bar{n}} v_{i}^{2}=\operatorname{Min}, \quad i=1,2, \ldots, \bar{n}
$$

werden soll.

Hat man nun eine Reihe zusammengehöriger Wertepaare $L_{i}, L_{i}^{\prime}$ gemessen, und soll aus theoretischen Gründen zwischen den Meßgrößen und den gesuchten Konstanten $x, y, \ldots$ eine bestimmte Relation bestehen, so werde dies ganz allgemein durch

$$
f_{i}\left(L_{i}+v_{i}, L_{i}^{\prime}+v_{i}^{\prime}, x, y, \ldots\right)=0
$$

ausgedrückt.

Hierbei erscheinen die $L$ bzw. $L^{\prime}$ um $v$ bzw. $v^{\prime}$ verbessert, da man aus den genannten Gründen von den reinen Meßzahlen $L$ und $L^{\prime}$ wird nicht erwarten können, daß sie die in Rede stehende Beziehung $f$ widerspruchsfrei erfüllen werden.

Es läßt sich nun zeigen, daß man statt dessen auch mit Funktionsverbesserungen $\lambda_{i}$ rechnen kann, so daß

$$
f_{i}\left(L_{i}, L_{i}^{\prime}, x, y, \ldots\right)-\lambda_{i}=0
$$

Ist $f$ ursprünglich eine nicht-lineare Funktion, so führe man Näherungswerte $x_{0} y_{0}, \ldots$ für die gesuchten Unbekannten ein, so daß

$$
x=x_{0}+\Delta x, \quad y=y_{0}+\Delta y, \ldots .
$$

Nach dem Satz von T a y lor erhält man unter Vernachlässigung der Glieder zweiter und höherer Ordnung

$$
\lambda_{i}=a_{i} \Delta x+b_{i} \Delta y+\ldots+l_{i},
$$

worin

$$
a_{i}=\frac{\partial f_{i}}{\partial x}, \quad b_{i}=\frac{\partial f_{i}}{\partial y}, \quad l_{i}=f_{i}\left(L_{i}, L_{i}^{\prime}, x_{0}, y_{0} \ldots\right) .
$$

Infolge des vorgenommenen Überganges von den wirklichen Verbesserungen $v_{i}$ und $v_{i}^{\prime}$ zu den Funktionsverbesserungen $\lambda_{i}$ muß jedoch jeder der „Fehlergleichungen“ (4) noch ein "Rechnungsgewicht“ $p_{i}$ zugeordnet werden, wobei

$$
\frac{\text { const }}{p_{i}}=\left(\frac{\partial f_{i}}{\partial L_{i}} m_{i}\right)^{2}+\left(\frac{\partial f_{i}}{\partial L_{i}{ }^{\prime}} m_{i}{ }^{\prime}\right)^{2},
$$

* Bamberg, Kunigundendamm 11. 